\title{
Bulges vs elliptical galaxies: some clues from their formation in a cosmological context
}

\author{
R. Domínguez-Tenreiro ${ }^{1}$, F. J. Martínez-Serrano ${ }^{2}$, A. Serna ${ }^{2}$, \\ M. Mollá ${ }^{3}$ and J. Oñorbe ${ }^{1}$ \\ ${ }^{1}$ Department of Theoretical Physics, Universidad Autónoma de Madrid, 28049 Madrid, Spain \\ email: rosa.dominguez@uam.es \\ ${ }^{2}$ Department of Physics and C.A., Universidad Miguel Hernández, 03206 Elche, Spain \\ ${ }^{3}$ Department of Fundamental Research, CIEMAT, 28040 Madrid, Spain
}

\begin{abstract}
We report on an extension, through cosmological simulations, of the two-phase formation scenario for elliptical galaxies to classical spiral bulges. In particular, we analyze the possibility that the old stellar population of bulges forms in a fast phase, while the younger stellar component forms or is assembled in a slow phase, in part from disk material suffering instabilities and in part through satellite capture or mergers.
\end{abstract}

Keywords. methods: hydrodynamics, galaxies: formation, galaxies: bulges, stars: formation

\section{Introduction}

Elliptical galaxies and the bulges of spirals show similarities and differences from the point of view of observational data, and, in fact, a longstanding matter of debate in Astronomy is how far the analogies between bulges and ellipticals go (see, for example, Wyse, Gilmore \& Franx 1997; Ellis, Abraham \& Dickinson 2001). In this paper we address this issue focusing on the formation of their stellar populations (SP).

Results of hydrodynamical simulations in a cosmological context indicate that two different phases operate in the acquisition of baryons by the elliptical-like-objects (hereafter, ELOs) formed in the simulations: first, a fast one, where the merging rate is high and most stars are formed; then, a slower one, with lower merging rates and where dry mergers are responsible for most of the mass increase. This scenario nicely explains the structural and dynamical properties of elliptical galaxies (Oñorbe et al. 2005; Domínguez-Tenreiro et al. 2006), as well as the age effects in their stellar populations (Domínguez-Tenreiro, Sáiz, \& Serna 2004 and refererences therein). In this work we report on preliminary results suggesting that bulge mass assembly can also be described as a two-phase process, where the difference mainly rests on the respective timescales for star formation (SF).

\section{Halo \& ELO formation}

The two-phase scenario for elliptical formation reflects the mass assembly history of their dark halos. In fact, analytical models, as well as N-body simulations, show that two different phases operate along halo mass assembly, first, a violent fast one, and then, a slower one, with high and lower mass aggregation rates, respectively (Wechsler et al. 2002; Zhao, Mo, Jing \& Borner 2003; Salvador-Solé, Manrique, \& Solanes 2005). In simulations, it is found that these patterns of formation are common to dark haloes hosting both ELOs or disk-like-objects (DLOs, i.e., virtual spirals).

Concerning ELOs, our simulations show (Domínguez-Tenreiro et al. 2006) that they assembled out of the mass elements that at high $z$ are enclosed by those overdense regions 
$\mathrm{R}$ enclosing a mass of the order of an E galaxy virial mass and whose local merging activity is much higher than average. These overdense regions act as mass flow convergence regions (FCRs hereafter), whose baryon content defines the particles that will end up in a bound configuration forming an ELO. They first expand slower than average, then they turn around and collapse through fast global compressions, involving the cellular structure elements they enclose and in particular nodes connected by filaments, that experience fast head-on fusions (i.e., multiclump collapse, see Thomas, Greggio \& Bender 1999). This results in strong and very fast SF bursts at disconnected locations, that transform most of the available cold gas at the FCR into stars, so that most of the dissipation and the SF involved in the mass assembly of a given ELO occurs in this early phase (between $z \sim 6$ and $z \sim 2.5$ for massive ELOs) and mainly corresponds to a cold mode of gas aggregation (as in Keres et al. 2005) through filaments. After this fast phase, our simulations indicate that the merger rate considerably decreases and that mergers imply only a modest amount of energy dissipation or star formation. However, the stellar mass increment through mergers in the slow phase is, on average, very important.

\section{Two-phase bulge formation?}

To answer to the question of how far can we extend the analogies between the assembly patterns of ellipticals and (classical) bulges, we have simulated the evolution of a $10 \mathrm{Mpc}$ side periodic box within a concordance model using the P-DEVA code (for details see Serna, Domínguez-Tenreiro \& Sáiz 2003 and Serna et al. in preparation), including a probabilistic self-consistent implementation of chemical evolution (see Martínez-Serrano et al. these proceedings), that implies metal enrichment of the SPs and the presence of metal-enriched gas-again particles coming from their passive evolution. Virtual galaxies of different morphological types form in the simulations. DLOs have been identified as those virtual galaxies having a populated, extended disk, and, for the purposes of this work, a central spheroidal or classical bulge-like component. They have been resimulated at higher resolution using a multimass technique. Here we present preliminary results of an analysis of three of them. The softening and number of baryon particles in these high resolution simulations are $(550,300,300)$ pc and $(15551,29167,42609)$, respectively, corresponding to stellar masses of $(4.5,1.5,1.0) \times 10^{10} \mathrm{M}_{\odot}$ per DLO.

We have followed the mass assembly of these three bulges. We describe what we have witnessed, focusing on when and where their stars form: i) First, a fast multiclump collapse phase, similar to that described above for ELOs, except that the mass enclosed by the FCR is lower and that relatively more gas is left at the FCR when this phase is over. As a consequence, a first stellar population forms in the densest clumps, placed at disconnected locations within the FCR, at high $z$ and on short timescales. ii) After that, a slow phase comes into play. The assembly patterns differ from those in ELO assembly mainly in that dissipation is relevant in bulge formation during the slow phase. In fact, gaseous disks are grown from the gas left at the FCR, then these mass elements can possibly be turned into stars, and then standard disk-driven processess of inward mass transport (Kormendy \& Kennicutt 2004; Carollo et al. 2007) feed the bulge with younger stellar populations, causing its rejuvenation. At the same time, more direct bulge feeding processes are possible (i.e., satellite capture, Eliche del Moral et al. 2006), and, moreover, the passive evolution of the stellar population formed in the fast phase could provide metal-enriched and $\alpha$-enhanced material to form succesive stellar generations. As an illustration of the different phases of SF, in figure 1 we plot the resulting SF rate history corresponding to those stars that form, at $z=0$, the bulge of one of the DLOs (note the similarity with the SFRHs Mollá, Ferrini \& Gozzi 2000 and Ferreras, 


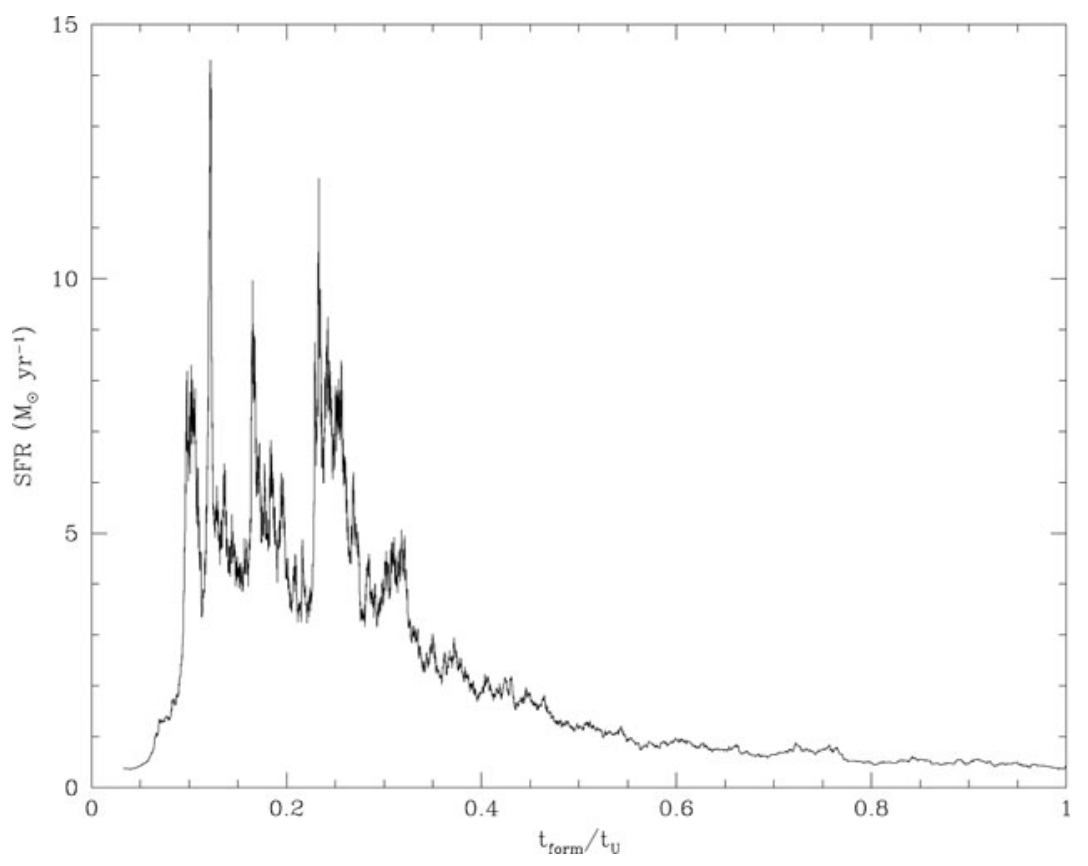

Figure 1. The SF rate history of the stars forming the bulge of a typical DLO at $z=0$

Wyse \& Silk 2003 propound in their models). The effects of turn-around on this SF rate can be clearly appreciated in this figure at $t_{\text {form }} / t_{\mathrm{U}} \sim 0.32(z=1.5)$, a rough frontier between the fast and the slow phase of mass aggregation. Accordingly, the stellar particles forming the bulge at $z=0$ can be classified as O-type or Y-type particles, depending on whether their transformation into stars occurred during the fast or the slow phases, respectively. To deepen into the relationship between mass assembly and SF, we have separately followed their assembly history to form the bulge.

At large scales, we have found that at any $z$ during the fast phase, the two populations sample a filamentary structure suffering a clumpy collapse, but they are spatially segregated. The densest sub-volumes of the FCR are sampled by the mass elements corresponding to the old population, until their complete merging at $z=1.5$ to form a spheroidal component, while most of the Y-type particles are at this moment still far from their final location at the bulge. In fact, these Y-type particles will form part of the disk component before they reach the bulge at the slow phase. Looking at smaller scales, we have found that part of the Y-type mass elements belong to the disk also during the fast phase. At $z=0$, there is an age gradient, with younger populations and still some $\mathrm{SF}$ at the center.

Another important issue is the metallicity distribution in bulges. Resolved stellar populations in bulges show that they are metal rich in the mean, with a broad distribution (see Minniti and Arimoto, these proceedings, and references therein). This is just what we have found here. Moreover, the different assembly patterns of $\mathrm{O}$ - and Y-type baryon particles is also reflected by their chemical composition. Our simulations indicate that the former are generally more metal-poor and somewhat more $\alpha$-element enhanceded than the later. This explains the observed gradients (Jablonka, Gorgas \& Goudfrooij 2007), with older ages, lower metallicities and higher $\alpha$-element enhancement at the outer bulge regions. For further details see Martínez-Serrano et al., these proceedings. 


\section{Discussion}

We see that our simulations provide a scenario where many properties of classical bulge SPs, as inferred from observations, can be explained. The fast phase is basically an early collapse, close the Eggen, Lynden-Bell \& Sandage (1962) phenomenological model (but not monolithic or spherical), and can explain metallicity, age and kinematics correlations, and the slow phase can involve secular effects (Kormendy \& Kennicutt 2004; Athanassoula 2005) linking some disk and bulge properties. We have found that mergers are more frequent, and, in some cases, multiple, in the fast phase, but some mergers can also occur during the slow phase, depending on environment, and trigger inward mass transport. Two-phase formation scenarios had already been propounded, on a phenomenological basis, by other authors (see for example, Ellis, Abraham \& Dickinson 2000; Prugniel, Maubon \& Simien 2001; Thomas \& Davies 2006), see also Gibson, Kowayashi and Steinmetz, these proceedings, and their quoted references. We additionally note that pseudobulges could also be accomodated in this scenario (provided that they host small classical bulges, as found in NGC 2950, Kormedy \& Barentine in preparation), as the central parts of disk galaxies living in low density environments that have never suffered a major merger since their formation (see Drory \& Fisher 2007), and, consequently, where Y-type population characteristics dominate over O-type ones.

Our main contribution to this debate is to show, through hydrodynamical simulations in a cosmological context, that this two-phase scenario naturally results from simple physical laws acting on initial conditions that are realizations of power spectra consistent with observations of CMB anisotropies.

\section{Acknowledgements}

We are indebted to the CCCFC (UAM) for computing facilities and to the MCyT (Spain) and the regional government of Madrid for financial support through grants AYA15492-C03 and the ASTROCAM Astrophysics network (S-0505/ESP-0237), respectively.

\section{References}

Athanassoula, E. 2005, MNRAS 358, 1477

Carollo, C. M., Scarlata, C., Stiavelli, M., Wyse, R. F. G., \& Mayer, L. 2007, ApJ 658, 960

Domínguez-Tenreiro, R., Sáiz, A., \& Serna, A. 2004, ApJ 611, L5

Domínguez-Tenreiro R., Oñorbe J., Sáiz A., Artal H., Serna A. 2006, ApJ 636, L77

Drory, N. \& Fisher, D- B. 2007, ApJ 664, 640

Eggen, O.J., Lynden-Bell, D., \& Sandage, A. 1962, ApJ 136, 749

Eliche-Moral, M. C., Balcells, M., Aguerri, J. \& González-García, A. C. 2006, A\&A A 457, 91

Ellis, R. S., Abraham, R. G. \& Dickinson, M. E. 2001, ApJ 551, 111

Ferreras, I., Wyse R. F. G. \& Silk, J. 2003, MNRAS 345, 1381

Jablonka, P., Gorgas, J. \& Goudfrooij, P. 2007, A\&A in press

Keres, D., Katz, N., Weinberg, D., \& Davé, R. 2005, MNRAS 363, 2

Kormendy, J. \& Kennicutt, R. C. 2004, ARAA 42, 603

Mollá, M., Ferrini, F. \& Gozzi, G. 2000, MNRAS 316, 345

Oñorbe, J., Domínguez-Tenreiro, R., Sáiz, A., Serna, A., \& Artal, H. 2005, ApJ 632, L570

Salvador-Solé, E., Manrique, A., \& Solanes, J. M. 2005, MNRAS 358, 901

Serna, A., Domínguez-Tenreiro, R. \& Sáiz, A. 2003, ApJ 597, 878

Thomas D., Greggio L. \& Bender R. 1999, MNRAS 302, 537

Wechsler, R. H. et al. 2002, ApJ 568, 52

Wyse, R. F. G., Gilmore, G. \& Franx, M. 1997, ARAA 35, 637

Zhao, D. H., Mo, H. J., Jing, Y. P., \& Borner, G. 2003, MNRAS 339, 127 